\title{
Critical Care Nurses' Involvement, Feeling, Perception and Barrier for Practice Breaking Bad News in Jordan
}

\author{
Yazeed M.Alkhawaldeh", Salam M.Abufeddeh, Khaled Izzeldeen \\ School of Nursing, University of Jordan, Amman, Jordan \\ *Corresponding author: Yazeed90alkhawaldeh@yahoo.com
}

\begin{abstract}
Background. Breaking bad news (BBN) is one of the most difficult tasks faced by healthcare providers. In addition, breaking bad news is stressful for both; healthcare providers and patient. Nurses can play an important role in the process of BBN. Whereas, effective communication by critical care nurses improves patients' understanding of their health status and decreases their stress level. Furthermore, effective BBN process requires effective communication skills. Aims. To assess critical care nurses' knowledge level regarding breaking bad news process, assess their involvement in the process of BBN, andto determine the barrier of practicing breaking bad news process as perceived by critical care nurses to identify the factors that affect nurses' practice of breaking bad news in the critical care units. Methods. Descriptive cross-sectional design, using self-reported questionnaires. Results. The study showed that most critical care nurses in Jordan are highly involved in BBN process $77 \%$ ( $n=157)$, Also, nurses are highly perceived themselves as an active part in $\mathrm{BBN}$ process $76 \%(\mathrm{n}=155)$. The major barrier in providing BBN was relatives requesting that a patient is not told bad news $75 \%(n=152)$. Conclusion. Current study revealed that Jordanian critical care nurses took different roles in the process of breaking bad news and they are highly involved in BBN news, creating a base of knowledge regarding BBN among critical care nurses in Jordan. Thus, the study recommends the policymakers to empower nurses role in this process through design an educational program regarding practice BBN and clarify nurses' role in this process.
\end{abstract}

Keywords: critical care, nurses, perception, barrier, breaking bad news

Cite This Article: Yazeed M.Alkhawaldeh, Salam M.Abufeddeh, and Khalid Ezzelden, "Critical Care Nurses' Involvement, Feeling, Perception and Barrier for Practice Breaking Bad News in Jordan." American Journal of Nursing Research, vol. 6, no. 3 (2018): 87-93. doi: 10.12691/ajnr-6-3-2.

\section{Introduction}

Breaking bad news (BBN) is one of the most difficult tasks faced by healthcare providers. Also, breaking bad news is stressful for both; healthcare provider and patient [1]. Critical care unit differs from other hospital departments because of the complexity of patient health problems, treatment modalities used [2], the nature of the environment, diversity of procedure and equipment [3], facing death and dying patients [4], and emotional needs of patients and their families [5]. Critical care units' patients and their families are suffering from high level of stress, anxiety and emotional exhaustion [6,7]. Therefore, the chance for deterioration ofpatients health status is very high which leads to more bad news that adds more stress and anxiety for them.

The first researcher that defined bad news was Buckman [8]. According to him, anything that significantly and negatively affects the attitude of a person toward their future is called bad news. A frequently cited definition of bad news in health care is that it is "any bad, sad or significant information that alters negatively people's expectations or perceptions of their present or future" [9]. Many definitions for breaking bad news in the literature were found in another way such that of Warnock [10], which stated that breaking of bad news is regarded to the time when a doctor and nurse sat down with a patient, family members, and any relative to patient to provide detailed information about a life-limiting diagnosis or poor prognosis. From the above definitions, we can highlight the importance of the role of the nurse in communicating bad news and conclude that breaking bad news is not only one event.

There are many reasons why nurses have difficulties during BBN process. The most important one is the unclear role for nurses involvement in BBN process [11]. Most sources in literature considered that physicians are responsible for providing it, while nurses play secondary role in assessingpatients' needs and clarifying misunderstood information.Literature showed that BBN process is considered as one of the doctors' responsibilities as mentioned by Mishelmovich, Arber and Odelius [12]. Also, Abbaszadeh and Ehsani [13] reported that providing bad news is one of physicians' responsibilities, while, nurses play an assisting role during the process of providing it by doctors $[10,14]$. Literature revealed many role for nurses during BBN process including providing information and helping patients prepare for receive, understand and cope with the bad news that they may receive [15].

Nurses can play an important role in the process of BBN. Whereas, effective communication by critical care 
nurses improve patients' understanding of their health status and decrease their stress level [6]. Furthermore, Warnock, Tod, Foster and Soreny [10] stated that nurses can support the patients by assessing, identifying and clarifying misunderstandings.

Nurses also have their own issues about breaking bad news. Previous researches were focused on the role and experiences of doctors when delivering bad news, which reflect their central role as providers of information about prognosis, diagnosis and treatment [16]. Several studies have clarifieda step-by-step guidance for each stage of the process of breaking bad news. These guidelines based on an interaction where there is an opportunity to prepare for give information in a relatively and some of them suggest that doctor's concentration at the moment of breaking bad news may be too narrow, in terms of understanding the patient's experience after receiving this news [17,18]. Literature shows that there is a lack of knowledge regarding $\mathrm{BBN}$ in the nursing field. Imanipour, Karim and Bahrani [19] stated that only $16.2 \%$ of critical care nurses had a good level of knowledge regarding BBN process. Inversely, nurses had positive attitude toward involvement on BBN process. Imanipour et al. [19] revealed that 91.2\% (among who?) positive attitude toward involvement in the BBN.

Effective BBN process requires effective communication skills [20], expressing empathy, and trust [21]. Pereira, Calônego, Lemonica, and Barros [22] mentioned that communication-training program should be added to the curriculum of each organization before entering the clinical field. Furthermore, they suggest preparing of healthcare providers through training programs that contain guidance for cognitive and behavioral coping strategies that can deal with any adverse reactions, which may came from patients or their families [23].

Literature showed that the major barrier for breaking bad news is the ineffective communication skills. Abbaszadeh and Ehsani [13] stated that weak communication could worsen misunderstanding of information, causing high stress level. In addition, Lin et al. [24] reported that the lack of communication training negatively affects nurses' perspective during communication with patients. Furthermore, Warnock, Buchanan and Tod [25] concluded that patients' diagnosis and treatment modalities could affect BBN process.

This current study, which is conducted in Jordan, has created a base for a sold body of knowledge regarding critical care nurses involvement, feeling and barriers in regard to $\mathrm{BBN}$ process. Also, it is considered the first study dealing with such topic in Jordan.

Examining the level of nurses' involvement and feeling perceived of BBN process has led to empowerment of nurses' role in this process as well [26].

Searching electronic data bases revealed no studies were conducted in Jordan and worldwide regarding assessment of critical care nurses' knowledge level, involvement and perceiving of barriers for BBN process. One study was found concerning the assessment of nurses' knowledge, involvement and barriers that they receive, as they perceive breaking bad news. It is expected that this study will create base of sold knowledge regarding critical care nurses' knowledge, practicing and perceiving barrier for BBN process. Furthermore, this first study conducted in Jordan regarding this topic. This study determines the need for improving such important role to improve nurses' knowledge, involvement in practice $\mathrm{BBN}$ process. In addition, it may help decrease the barriers involved in the process, leading to decrease anxiety, stress and emotional exhaustion among patients and their families.

The aims of this study are to assess critical care nurses' involvement level in breaking bad news process, assess their feeling and perception as a part in BBN process, and to determine the barrier of practicing breaking bad news process as perceived by critical care nurses to identify the factors that affect nurses' practice of breaking bad news in the critical care units. To achieve these aims, the study must answer the following questions:

1. What is the frequency of critical care nurses involvement in the practice of breaking bad news process?

2. How do critical care nurses perceive breaking bad news process?

3. What are the barriers of breaking bad news process as perceived by critical care nurses?

4. Are there statistically significant differences in the critical care nurses' practice of breaking bad news, and how they perceive the process based on their demographics?

\section{Methodology}

\subsection{Design}

This study had used a quantitative descriptive crosssectional design that carried out by using self-reported questionnaires to describe the level of nurses' practicing, feeling and perception of breaking bad news process at critical care units. In addition to using a questionnaire that describes the barriers as perceived by them.

\subsection{Setting}

The current study was conducted in critical care units at Jordan. Healthcare system in Jordan consists of four major sectors: private, military, governmental and educational. Military healthcare sector was excluded because data collection is mind by their policies. Hence, data were collected from three hospitals, representing different healthcare sectors in the middle region of Jordan. According to statistics of Ministry of Health regarding hospitals in Jordan (2012), the total number of hospitals in Jordan is 106 hospitals and the majority of hospitals (63\%) are located in the middle region. The total number of bed in these hospitals is 12106 beds. While bed capacity in the middle region 7627 beds. Hospitals was randomly selected from a list of hospitals in each sector and convenient sample was recruited from all critical care units inside the selected hospital.

\subsection{Sampling}

The target population of the study included all critical care nurses working in Jordanian hospitals, whereas accessible population included all critical care nurses working in the participating hospitals. Inclusion criteria 
included being a registered nurse holding at least bachelor degree in nursing and had an experience of at least six months in the critical care units. Exclusion criteria included administrative nurses who provide no direct patient care and nurses who have physical disabilities.

Applying multiple regression with moderate effect size 0.15 , power of 0.80 , and level of significance of 0.05 . The calculation yielded that a minimum of 107 nurses would be needed. However, a larger sample size was targeted to obtain more representative sample and to compensate for incomplete questionnaires.

\subsection{Data Collection Procedure}

Soon after getting the ethical approval, the researchers visited the nursing administration of the participating hospitals to facilitate meeting with critical care units' head nurses who facilitated approaching nurses in critical care units in an appropriate time. This was scheduled with head nurses and staff nurses to decrease work interruption. All nurses who met the inclusion criteria were invited to participate in this study. Explanation by the researchers for all eligible staff nurses had been given regarding the purpose of the study; the time that required to fill the questionnaire, which was between 10-15 minutes; and participant rights.

A questionnaire was given to each nurse who agreed verbally to participate in the study. They are asked to fill the questionnaire and to write their signature in the cover letter to assure their acceptance. The researcher was available during filling of the questionnaire to clarify and answer questions of the participants. After filling the questionnaires by nurses, the researcher collected the questionnaires and thanked participants for their cooperation and contribution to this study.

\subsection{Ethical Consideration}

The ethical approval for this study was gained from the Scientific Research Committee at School of Nursing at the university of Jordan and the participating hospitals. All ethical concepts was taken in consideration including maintaining participants' privacy and confidentiality, explaining the purpose and nature of the study, using an informed consent for the participants who voluntarily accepted to participate in the study without coercion, and exposing the participants them to no harm or risk.

\subsection{Instrument}

The study instrument package contained four sections: demographics, frequency of involvement in breaking bad news activity(Breaking bad news) tool, nurses feeling and perception in relation to the process of $\mathrm{BBN}$ tool, and Nurses perception barriers of BBN tool.

Demographics data sheet had been developed by the researchers after reviewing the literature, and it included gender, age, education level, area of critical care unit (surgical, medical, mixed and coronary care unit), and years of experience.

The frequency of critical care nurses involvement in breaking bad news was measured using nurses feeling and experience in relation to the process of BBN tool [27]. Which consists of seven items. Each of the seven items was rated by the respondent on a 3 - point Likert scale ranging from (3) Being all the time to (1) rarely. The total score was calculated with a possible range of responses between 7 to 2 with high scores indicating more involvement in $\mathrm{BBN}$ practice.

Critical care nurses perception and feeling toward BBN process is measured using Nurses feeling and perception in relation to the process of $\mathrm{BBN}$ tool [27]. It consists of twelve items; with each item rated by the respondent on 3point Likert scale, ranging from (3) Agree to (1) Disagree. The total score was calculated with a possible range of responses between 12 to 36, with higher scores indicating better perception and feeling in BBN practice.

The barriers as perceived by critical care nurses were measured using nurses perceive to barrier of BBN tool [28]. It is consists of nine items each of them is rated by the respondent on a 3-point Likert scale, ranging between (3) Being all the time and (1) rarely. The total score was calculated with a possible range of responses between 9 and 27; higher scoring indicate more barriers.

\subsection{Data Analysis Plan}

The statistical package for social science software (SPSS) (version 21) (Version 21.0; SPSS, Inc., Chicago, IL)had been used for data analysis. After cleaning the data, descriptive statistics were used to answer the first, second and third questions (mean, standard deviation and frequencies) to determine the frequency of nurses involvement in breaking bad news activity (Practice), level of nurses' feeling and experience in relation to the process of breaking bad news $(\mathrm{BBN})$, and barrier of breaking bad news as perceived by nurses. The fourth question had been answered using t-test and one-way analysis of variance (ANOVA) to determine the differences in main study variables based on their demographic characteristics.

\section{Results}

The number of nurses who met the inclusion criteria was 250 participants. Response rate were $81 \%(n=203)$. Socio-demographics of the participants are presented in Table 1 . The majority of participants were male $52.2 \%$ $(\mathrm{n}=106)$. Most of participants are aging from 26-30 years, which represents $53.6 \%(\mathrm{n}=109)$. In addition, only $15.2 \%$ of participants are holding higher educational degree. Private hospitals were the most frequent in the study, accounting for $36.5 \%(n=74)$. Also, mixed intensive care units (Mixed ICU) had the highest participant ratio $66 \%(n=134)$. Furthermore, most of participants are newly- graduated with works' experience ranges between 1 to 3 years $45.3 \%(n=92)$.

The average mean of involvement in $\mathrm{BBN}$ process is 2.3 out of 3 . The results indicated that most of participants are involved at all of the time in providing support to a patient/ relative following the breaking of bad news, with frequency level of 136. In contrast, only five participants reported that they are rarely involved in such activity. The 
total mean for this item is 2.65 (.529) see (Table 2). In addition, variations are noticed among the results for the item that focused on providing the patient/relative with opportunities to talk about the bad news. The number of participants who are involved most of the time in this activity was 104 , while only 16 participants were rarely involved in it. The remaining of participants indicated that they are involved sometimes in this activity with $\mathrm{f}$ level equal to 83 .

Nurses' feelings and experiences in relation to the process of $\mathrm{BBN}$ revealed that most of the participants are agreed about their ability in supporting patients and their relatives from different cultural backgrounds in relation to the process of breaking bad news, with frequency level 129. Most of them also agreed that being involved in the process of $\mathrm{BBN}$ can be rewarding as it can help the patient/relative prepare for the future, with frequency level of 123, and most of them agreed that they have good strategies for coping with their emotional reactions when involved in the process of BBN. In contrast, only 13 participants disagreed with the notion that their involvement in the process of BBN has strengthened their relationship with a patient/relative, which is the lowest recorded result.
Table 1. Demographic profile of the participants

\begin{tabular}{lc}
\hline Variable & Frequency (Percentage) \\
\hline Gender & $106(52.2)$ \\
Male & $97(47.8)$ \\
Female & \\
Area of working & $18(8.9)$ \\
SICU & $15(7.4)$ \\
MICU & $36(17.7)$ \\
CCU & $134(66.0)$ \\
Mixed & \\
Educational level & $172(84.7)$ \\
Bachelor & $31(15.3)$ \\
Higher degree & \\
Age & $61(30.0)$ \\
$22-25$ & $109(53.6)$ \\
$26-30$ & $27(13.4)$ \\
$31-35$ & $6(3.0)$ \\
$<35$ & \\
Years of experience & $92(45.3)$ \\
$1-3$ & $70(34.4)$ \\
$4-6$ & $35(17.3)$ \\
$7-10$ & $6(3.0)$ \\
$<10$ & \\
Hospital & $74(36.5)$ \\
Private & $67(33)$ \\
Governmental & $62(30.5)$ \\
Educational hospital &
\end{tabular}

Table 2. Frequency of involvement in each breaking bad news activity in the past 3 months

\begin{tabular}{|c|c|c|c|c|c|}
\hline & \multirow{2}{*}{ Item } & \multirow{2}{*}{ Mean (SD) } & \multicolumn{3}{|c|}{ Frequency } \\
\hline & & & Rarely & Sometime & All the time \\
\hline 1. & Providing support to a patient/ relative following the breaking of bad news. & $2.65(.529)$ & $5(2.46)$ & $62(30.54)$ & $136(67.0)$ \\
\hline 2. & $\begin{array}{l}\text { Providing the patient/relative with opportunities to talk about the bad news } \\
\text { given to them. }\end{array}$ & $2.43(.637)$ & $16(7.88)$ & $83(40.89)$ & $104(51.23)$ \\
\hline 3. & $\begin{array}{l}\text { Helping patients/relatives come to terms with the implications of bad news over } \\
\text { time. }\end{array}$ & $2.44(.545)$ & $5(2.46)$ & $104(51.23)$ & $94(46.31)$ \\
\hline 4. & Being present when a doctor informs the patient / relative of bad news. & $2.35(.598)$ & $13(6.40)$ & $106(52.22)$ & $84(41.38)$ \\
\hline 5. & $\begin{array}{l}\text { Discussing bad news when the patient/relative asks questions on an ad hoc } \\
\text { basis. }\end{array}$ & $2.19(.671)$ & $30(14.80)$ & $105(51.70)$ & $86(33.50)$ \\
\hline 6. & Preparing patients/relatives for bad news. & $2.15(.75)$ & $44(21.68)$ & $85(41.87)$ & $74(36.45)$ \\
\hline \multirow[t]{2}{*}{7.} & Actually breaking bad news to a patient/relative. & $1.94(.65)$ & $50(24.63)$ & $116(18.23)$ & $37(57.14)$ \\
\hline & Average & $2.31(0.63)$ & & & \\
\hline
\end{tabular}

Table 3. Nurses' feelings and experiences in relation to the process of breaking bad news (BBN)

\begin{tabular}{|c|c|c|c|c|c|}
\hline & \multirow[b]{2}{*}{ Item } & \multirow[b]{2}{*}{$\operatorname{Mean}(\mathrm{SD})$} & \multicolumn{3}{|c|}{ Frequency } \\
\hline & & & Disagree & $\begin{array}{l}\text { Neither agree } \\
\text { nor disagree }\end{array}$ & Agree \\
\hline 1. & $\begin{array}{l}\text { Being involved in the process of } \mathrm{BBN} \text { can be rewarding as it can help the } \\
\text { patient/relative prepare for the future. }\end{array}$ & $2.48(.71)$ & $25(13.32)$ & 55 (27.09) & 123 (60.59) \\
\hline 2. & $\begin{array}{l}\text { Being involved in the process of } \mathrm{BBN} \text { has strengthened my relationship with a } \\
\text { patient/relative. }\end{array}$ & $2.52(.62)$ & $13(6.40)$ & 72 (36.47) & $118(58.13)$ \\
\hline 3. & $\begin{array}{l}\text { Being involved in the process of } \mathrm{BBN} \text { has encouraged me to reflect positively on } \\
\text { my own priorities and what is important in life. }\end{array}$ & $2.44(.70)$ & $24(11.82)$ & $65(32.02)$ & $114(56.16)$ \\
\hline 4. & $\begin{array}{l}\text { Being involved in the process of BBN has allowed me to share in important life } \\
\text { changing moments with patients/relatives. }\end{array}$ & $2.35(.68)$ & $23(11.33)$ & $86(42.36)$ & $94(46.31)$ \\
\hline 5. & I feel able to initiate discussions with patient/ relatives relating to bad news. & $2.39(.71)$ & $26(12.81)$ & $71(34.98)$ & $106(52.21)$ \\
\hline 6. & $\begin{array}{l}\text { I have good strategies for coping with my emotional reactions when involved in } \\
\text { the process of BBN. }\end{array}$ & $2.51(.67)$ & $20(9.85)$ & $60(29.56)$ & $123(60.59)$ \\
\hline 7. & I feel confident in my skills in relation to the process of breaking bad news. & $2.45(.68)$ & $22(10.84)$ & $68(33.50)$ & $113(55.66)$ \\
\hline 8. & $\begin{array}{l}\text { The area I work in has a good system of support for nurses involved in the } \\
\text { process of BBN. }\end{array}$ & $2.23(.75)$ & $39(19.21)$ & $78(38.42)$ & $86(42.37)$ \\
\hline 9. & $\begin{array}{l}\text { I feel able to support patients and relatives from different cultural backgrounds in } \\
\text { relation to the process of breaking bad news }\end{array}$ & $2.55(.65)$ & $18(8.87)$ & $56(27.58)$ & $129(63.55)$ \\
\hline 10. & I find it difficult to deal with patient's/relative's emotional reactions to bad news. & $2.03(.81)$ & $64(31.53)$ & $69(33.99)$ & $70(34.48)$ \\
\hline 11. & $\begin{array}{l}\text { Being involved in the process of BBN has had a negative effect on my } \\
\text { relationship with a patient/relative. }\end{array}$ & $1.87(.82)$ & $83(40.89)$ & $64(31.53)$ & $56(27.58)$ \\
\hline \multirow[t]{2}{*}{12.} & I try to avoid being involved in BBN as I find it difficult. & $1.83(.83)$ & $90(44.33)$ & $58(28.57)$ & $55(27.10)$ \\
\hline & Average score & $2.3(.72)$ & & & \\
\hline
\end{tabular}


Table 4. Frequency of encountering specific barriers to breaking bad news

\begin{tabular}{|c|c|c|c|c|c|}
\hline & \multirow{2}{*}{ Item } & \multicolumn{3}{|c|}{ Mean (Std) } & \multirow{2}{*}{$\begin{array}{l}\text { Frequency } \\
\text { All the time }\end{array}$} \\
\hline & & & Rarely & Sometime & \\
\hline 1. & Not having time to do it properly. & $2.19(.60)$ & $21(10.35)$ & $122(60.10)$ & $60(29.55)$ \\
\hline 2. & The patient not wanting to know. & $1.85(.73)$ & $72(35.47)$ & $90(44.33)$ & $41(20.20)$ \\
\hline 3. & Not feeling prepared as the issue was raised unexpectedly. & $1.98(68)$ & $49(24.14)$ & $109(53.69)$ & $45(22.17)$ \\
\hline 4. & Barriers to communication (e.g. language, dysphasia). & $1.71(.69)$ & $86(42.36)$ & $89(43.84)$ & $28(13.80)$ \\
\hline 5. & Not having enough information. & $1.90(.61)$ & $49(24.14)$ & $125(61.58)$ & $29(14.28)$ \\
\hline 7. & Lack of privacy. & $1.89(.77)$ & $73(35.96)$ & $80(39.41)$ & $50(24.63)$ \\
\hline 8. & Verbal or physical abuse from the patient/relative. & $1.95(.65)$ & $49(24.14)$ & $116(51.14)$ & $38(18.72)$ \\
\hline \multirow[t]{2}{*}{9.} & Nurses are not encouraged to be involved in breaking bad news in my area. & $2.07(.78)$ & $54(26.6)$ & $80(39.4)$ & $69(34.0)$ \\
\hline & Average score & $1.98(.67)$ & & & \\
\hline
\end{tabular}

The Average mean for the items is 2.3 out of 3 with percentage of $72 \%$ (see Table 3 ). In addition, the results for the item that focused on "Being involved in the process of $\mathrm{BBN}$ have had a negative effect on their relationship with a patient/relative "has a comparable results. The numbers of participants who are agreed and support this item was 56, while 83 participants show disagreement. The remaining of participants indicated that they are neither agreeing nor disagree for this item with $f$ level equal to 64 .

The frequency of encountering specific barriers to BBN showed that the most of participants are sometimes consider relatives requesting that a patient not to be told bad news as a barrier to breaking bad news, with frequency level of 128 . In contrast, only 10 participants reported that they are rarely faced the last same barrier. The total mean for this item is 2.27 (.55) (see Table 4). In addition, the results for the item that focused on not having time to do it properly has a comparable results. The number of participants who are all the time consider it as a barrier was 60 , while only 21 participants were rarely consider it as a barrier. The remaining of participants indicated that they are sometimes considering it as a barrier with $\mathrm{f}$ level equal to 122 . The average mean for these Items is 1.98 out of 3 with a percentage of $67 \%$.

Inferential statistics regarding the difference in nurses' practice of breaking bad news and how they are perceived the process of breaking bad news critical care units based on their demographics revealed that there are no statistically significant difference ( $p$ value $=0.06$, T test and ANOVA) between critical care nurses' practice of breaking bad news and how they perceive the process based on their demographics.

\section{Discussion}

The most difficult task faced by healthcare providers is BBN process. Furthermore, breaking bad news is stressful for both; nurse and patient [1]. Critical care setting defers from other health care setting because of complexity of patient health status and treatment modalities. Also, patients in critical care units experience high level of stress and multi comorbidity disease and may deteriorate any time leading to provide bad news and more stressors.

Nurses play major role in the breaking bad news process by using effective communication channels to improve patients' understanding of their health status, leading to decrease stress level [6]. Only $16 \%$ of critical care nurses have a good level of knowledge regarding breaking bad news [19]. Literature revealed that ineffective communication skills are considered the major barrier for breaking bad news process. Whereas, weak communication leads to misunderstanding the given information and leads to high stress level. Likewise, 50\% of patient in ICU complaining from inadequate communication with nurses [29]. The aim of this study was to assess critical care nursing knowledge, practice level, and barriers in breaking bad news process to enhance their role in this process.

Findings of this study regarding Jordanian critical care nurses revealed that $63 \%$ of nurses were involved in the practice of breaking bad news process. Imanipour et al. [19] reported that most of critical care nurses are involved in breaking bad news and most nurses (67\%) are involved in providing support to patient and patient relatives all the time but a few nurses are actually practice breaking bad news to patient and relatives (18\%). Similarly, a study by Warnock [10] stated that most of nurses are involved in providing support to patient and their relatives $(55.5 \%)$, and $(2.5 \%)$ of nurses are actually breaking bad news all the time. These studies confirm that nurses across different role are involved in breaking bad news, even if there is no responsibility is required.

As well, this study showed that $72 \%$ of nurses perceive themselves as an active part in breaking bad news. Similarly, Warnock et al. [10] reported that about $51 \%$ of nurses thought they are able to be an active part in breaking bad news. Most of nurses (64\%) feel that they are able to support patient and relatives from different cultural background in relation to breaking bad news process. Warnock et al. [10] showed that (42\%) feel that they are able to support patient and relatives from different cultural background in relation to breaking bad news process; this is because of differences in context of culture, nurses' duties, and organization policies. Furthermore, a few nurses (27\%) try to avoid being involved in breaking bad news. This result confirmed by Warnock et al. [10] showed that $(6 \%)$ of nurses try to avoid being involved in breaking bad news.

Literature revealed that a major barrier for delivering bad news is lack of nurses' knowledge [25,26]. In this study, $67 \%$ of Jordanian critical care nurses stated that they face barrier during breaking bad news process, in which major barrier to nurses $(73 \%)$ was not having time to do it. However, Warnock et al. [10] stated that the major barrier is lack of privacy in the area of practice, accounting for $22 \%$. The least barrier in this study was 
barrier of communication (language, dysphasia...etc.). Compared with Warnock et al. [10], the least one was Nurses are not encouraged to be involved in breaking bad news $(2 \%)$. The reason for this significant difference may due to different clinical settings that were studied, condition in critical care units, heavy workload, and context of nurses' role.

Regarding the differences in nurses' practice of BBN and how they perceive the process of BBN critical care units based on their demographics, the study revealed that there are no statistically significant differences between critical care nurses' practice of $\mathrm{BBN}$ and how they perceive the process based on their demographics. However, there is no studies in literature that test the deference between nurses' practice level and their perception of BBN process based on their demographics.

Study results revealed that nurses act major role in BBN process. Hence, it is important to empower nurses' role in this process by increasing body of knowledge and improving communication skills. Because that well prepared nurse; knowledge and communication skills help in improve patient understanding of their health status which help decrease stress level and enhance practicing $\mathrm{BBN}$ process. Also, to prevent the barrier of practice $\mathrm{BBN}$ process. Policy makers should pay more attention to nurses' role in regard to this process by building effective policies, designing training program for communication skills, improving nurse-patient relation to decrease workload because heavy workload and lack of time are considered within the major barrier as mentioned previously in the study.

\section{Conclusion}

Critical care units are considered as the most stressful units in the hospital due to their complexity. Patients in the critical care units have multi -comorbidities diseases and the probability for patient health status deterioration is high, leading to emergence of bad news. The process of BBN is stressful for nurses and patient. The current study showed new base of knowledge regarding critical care nurses involvement, feeling perceived by them, and barriers they face in BBN process. However, this study has some limitation that must be avoided in the future studies including a small sample size and adopting an inclusion criteria that only included critical care nurses in the middle region in Jordan. Hence, study recommends that future studies have to encompass all provinces in Jordan, and include military healthcare setting; and policy maker must be encouraged to develop training program and guidelines for critical care nurses regarding BBN process.

\section{References}

[1] Ozyemisci-Taskiran, O., Coskun, O., Budakoglu, I. I., \&Demirsoy, N. (2017). Breaking bad news in spinal cord injury; a qualitative study assessing the perspective of spinal cord injury survivors in Turkey. The Journal of Spinal Cord Medicine, 1-8.

[2] Köse, I., Zincircioğlu, C., Öztürk, Y., Çakmak, M., Güldoğan, E., Demir, H., ... \&Gonullu, M. (2016). Factors affecting anxiety and depression symptoms in relatives of intensive care unit patients. Journal of Intensive Care Medicine, 31(9), 611-617.
[3] Yava, A., Tosun, N., Ünver, V., \&Çiçek, H. (2011).Patient and nurse perceptions of stressors in the intensive care unit. Stress and Health, 27(2), e36-e47.

[4] Magnavita, N., \&Heponiemi, T. (2011). Workplace violence against nursing students and nurses: an Italian experience. Journal of Nursing Scholarship, 43(2), 203-210.

[5] Milutinović, D., Golubović, B., Brkić, N., \& Prokeš, B. (2012). Professional stress and health among critical care nurses in Serbia. Archives of Industrial Hygiene and Toxicology, 63(2), 171-180.

[6] Adams, A., Mannix, T., \& Harrington, A. (2015). Nurses' communication with families in the intensive care unit-a literature review. Nursing in Critical Care.

[7] Stayt, L. C. (2009). Death, empathy and self preservation: the emotional labour of caring for families of the critically ill in adult intensive care. Journal of Clinical Nursing, 18(9), 1267-1275.

[8] Lloyd-Williams, M. (2002). Breaking bad news to patients and relatives. Careerfocus, 325(7355), S-11.

[9] Bies, R. J. (2013). The delivery of bad news in organizations a framework for analysis. Journal of Management, 39(1), 136-162.

[10] Warnock, C., Tod, A., Foster, J., \& Soreny, C. (2010). Breaking bad news in inpatient clinical settings: role of the nurse. Journal of Advanced Nursing, 66(7), 1543-1555.

[11] Kirby, R. (2016). Breaking bad news. Trends in Urology \& Men's Health, 7(6), 4-4.

[12] Mishelmovich, N., Arber, A., \& Odelius, A. (2016).Breaking significant news: The experience of clinical nurse specialists in cancer and palliative care. European Journal of Oncology Nursing $21,153-159$.

[13] Abbaszadeh, A., \&Ehsani, S. (2014). Nurses' perspectives on breaking bad news to patients and their families: a qualitative content analysis. Journal of Medical Ethics and History of Medicine, 7.

[14] Warnock, C. (2014). Breaking bad news: issues relating to nursing practice. Nursing Standard, 28(45), 51-58.

[15] Tuffrey-Wijne, I. (2013). A new model for breaking bad news to people with intellectual disabilities. Palliative Medicine, 27(1), $5-12$.

[16] Schildmann, J., Kupfer, S., Burchardi, N., \& Vollmann, J. (2012). Teaching and evaluating breaking bad news: A pre-post evaluation study of a teaching intervention for medical students and a comparative analysis of different measurement instruments and raters. Patient education and counseling, 86(2), 210-219.

[17] Randall T. \& Wearn A. (2005) Receiving bad news: patients with haematological cancer reflect on their experiences. Palliative Medicine 19, 594-601

[18] Tobin G. \& Begley C. (2008) Receiving bad news. A phenomenological exploration of the lived experience of receiving a cancer diagnosis. Cancer Nursing 31(5), E31-E39.

[19] Imanipour, M., Karim, Z., \&Bahrani, N. (2016). Role, perspective and knowledge of Iranian critical care nurses about breaking bad news. Australian Critical Care, 29(2), 77-82

[20] Hemming, L. (2017). Breaking bad news: a case study on communication in health care. Gastrointestinal Nursing, 15(1), 43-50.

[21] Hyer, R., \& Covello, V. T. (2017). Breaking bad news in the highconcern, low trust setting: how to get your story heard. Health Physics, 112(2), 111-115.

[22] Pereira, C., Calônego, M., Lemonica, L., \& Barros, G. (2017). The PACIENTE Protocol: An instrument for breaking bad news adapted to the Brazilian medical reality. Revista da Associação Médica Brasileira, 63(1), 43-49.

[23] Ptacek, J. T., Fries, E. A., Eberhardt, T. L., \& Ptacek, J. J. (1999). Breaking bad news to patients: physicians' perceptions of the process. Supportive Care in Cancer, 7(3), 113-120.

[24] Lin, M., Hsu, W., Huang, M., Su, Y., Crawford, P., \& Tang, C. (2017). "I couldn't even talk to the patient": Barriers to communicating with cancer patients as perceived by nursing students. European Journal of Cancer Care.

[25] Warnock, C., Buchanan, J., \&Tod, A. (2017). The difficulties experienced by nurses and healthcare staff involved in the process of breaking bad news. Journal of Advanced Nursing.

[26] Arbabi, M., Rozdar, A., Taher, M., Shirzad, S., \& Ansari, S. (2013). Patients' preference to hear bad news. Psychotherapy \& Psychosomatics, 82, 7. 
[27] Palfreyman, S., Tod, A., \& Doyle, J. (2003). An integrated approach to evidence based practice. Foundation of Nursing Studies Dissemination Series, 9, 1-4.

[28] Tod, M., Bond, B., Leonard, N., Gilsenan, J., \&Palfreyman, S. (2007). Exploring the contribution of the Clinical Librarian to facilitating evidence - based nursing. Journal of Clinical Nursing, 16(4), 621-629.
[29] Schubart, J., Wojnar, M., Dillard, J., Meczkowski, E., Kanaskie, M., Blackall, G., ... \& Lloyd, T. (2015). ICU family communication and health care professionals: A qualitative analysis of perspectives. Intensive and Critical Care Nursing, 31(5), 315-321. 